\title{
Responses to flooding stress in soybean seedlings with the alcohol dehydrogenase transgene
}

\author{
Makoto Tougou, * Akiko Hashiguchi, Kiyoshi Yukawa, Yohei Nanjo, \\ Susumu Hiraga, Takuji Nakamura, Keito Nishizawa, Setsuko Komatsu \\ NARO Institute of Crop Science (NICS), Tsukuba, Ibaraki 305-8518, Japan \\ *E-mail: tougou@affrc.go.jp Tel \& Fax: +81-29-838-8392
}

Received November 24, 2011; accepted March 1, 2012 (Edited by T. Anai)

\begin{abstract}
The soybean is relatively sensitive to disturbances arising from flooding before its emergence from the soil. When young soybean seedlings at an early stage are transferred to flooding anaerobic conditions, alcohol dehydrogenase $(A d h)$ mRNA and Adh protein increase temporarily in the root tips, where active cell division demands high energy production. Since there is little information on the significance of the up-regulation of Adh for the tolerance of soybeans to flooding stress, we examined the response to flooding in transgenic soybean lines in which the soybean Adh (GmAdh2) gene was introduced under the control of a constitutive promoter. Acquired transgenic soybean seeds from one out of 14 transgenic lines were subjected to flooding stress. Growth inhibition of soybean seedlings caused by flooding stress was reduced in soybeans with the GmAdh2 transgene. Protein analysis and enzyme assay at the early stage of growth of the soybean seedlings confirmed that Adh expressions and activities in transgenic soybeans were increased compared to control soybeans. These results indicated that the introduced GmAdh2 gene might have induced some change in glycolysis and alcohol fermentation, and improved the germination of transgenic soybeans under flooding stress.
\end{abstract}

Key words: Alcohol dehydrogenase, flooding, soybean, transformation, transgenic soybean.

Soybeans are generally intolerant of flooding stress. In many regions of Japan, soybean seeds are sown in a paddy field during the summer-rainy season, and excess rainfall after sowing can often lead to soil flooding. Flooding after sowing causes severely decreased crop yields. These lower yields may result from the collapse of cotyledons due to rapid imbibitions of water (Nakayama et al. 2004) and from serious damage to the root system. Accordingly, it is important to understand the mechanism of the flooding stress responses in order to improve crop yields. However, the flooding stress responses in soybeans are not well characterized.

Studies of the responses of soybean seedlings to flooding stress showed that flooding inhibits root elongation and hypocotyl pigmentation (Hashiguchi et al. 2009) and affects the expression of some proteins involved in the processes of fermentation (Russell et al. 1990), the scavenging of reactive oxygen species (Shi et al. 2008), glycolysis, protein storage, and defense against disease (Hashiguchi et al. 2009). Since alcoholic fermentation is the major fermentation pathway of glycolysis in anaerobic plants (Rees et al. 1987; Komatsu et al. 2010a), the mechanism of tolerance to flooding stress should include the upregulation of genes engaged in glycolysis and alcohol fermentation. Under low oxygen stress conditions, plants activate alcohol fermentation in which pyruvate is used as a starting substrate. Pyruvate decarboxylase (Pdc, EC 4.1.1.1) catalyzes the first step and converts pyruvate to acetaldehyde. Then, alcohol dehydrogenase (Adh, EC 1.1.1.1) converts acetaldehyde to ethanol, with the concomitant regeneration of $\mathrm{NAD}^{+}$ for glycolysis. Adh is a fermentative enzyme that is highly conserved across species. Newman and VanToai (1992) described the soybean Adh genes that were expressed in anaerobic conditions. Oxygen deprivation acts as the prime signal in the plant's response to flooding (Jackson and Colmer 2005).

Recently, Komatsu et al. (2009, 2010a) and Nanjo et al. (2010) reported the results of proteome and transcriptome analyses of flooding-inducible proteins and genes, and showed that the expression of Adh increases remarkably in the early soybean growth stage as a result of flooding stress. Komatsu et al. (2010a, 2011) demonstrated that flooding induces a considerably greater upregulation of soybean Adh than of rice Adh, but the accumulation of soybean Adh decreased faster

Abbreviations: Adh, alcohol dehydrogenase; CaMV, cauliflower mosaic virus; hpt, hygromycin phosphotransferase, 2-DE, two-dimensional polyacrylamide gel electrophoresis; $\mathrm{CBB}$, coomassie brilliant blue; Pdc, pyruvate decarboxylase.

This article can be found at http://www.jspcmb.jp/

Published online May 30, 2012 
than that of rice after the drainage of the floodwater. A limiting concentration of pyruvic acid or a carbohydrate shortage could be caused by a shift to inefficient anaerobic carbohydrate catabolism in response to flooding. Rice, with its higher carbohydrate status, would need a relatively lower level of Adh to survive flooding than would soybeans, which contain an abundance of protein and oil. In this study, we produced transgenic soybeans that overexpress the GmAdh2 (Komatsu et al. 2011) gene, which was isolated from the soybean cultivar Enrei, and examined the responses of this transgenic soybean to flooding.

The GmAdh2 gene was amplified by PCR from the corresponding soybean (cv. Enrei) cDNA using a SMART PCR cDNA synthesis kit (Clonetech, USA). The forward primer was $5^{\prime}$-ATGTCGAGCACAGCT GGCCAAG- $3^{\prime}$. We used the $5^{\prime}$ SMART primer IIA CDS primer for the reverse primer. The $1.3-\mathrm{kb}$ amplified fragment was cloned into pMD20-T (Takara, Japan). Its sequence was almost identical ( $99.1 \%$ identity) to that from cv. Nourin No. 2 (DDBJ/GenBank/EMBL accession no. AK244789), and the protein product was almost identical (98.4\% identity) to that from cv. Williams 82 (phytozome database, Glyma04g41990.1). The cloned GmAdh2 gene fragment with each appropriate restriction enzyme recognition site $(\mathrm{XbaI}$ and $S a c I$ ) was inserted into pUC19, which contained the cauliflower mosaic virus (CaMV) $35 \mathrm{~S}$ promoter and NOS terminator. The resultant plasmid was named pCN-Adh2 (Figure 1). pE2113-HPT, which contained the hygromycin phosphotransferase $(h p t)$ gene, was used as a source of the selection marker gene, as described by Furutani and Hidaka (2004). We produced transgenic soybeans according to the protocols of Furutani and Hidaka (2004). The soybean (cv. Jack) was used for the induction and proliferation of somatic embryos. The embryos were co-transformed with pCN-Adh2 and pE2113-HPT using the PDS 1000/He particle gun (BioRad, USA). After the embryos had been selected with hygromycin, plants germinated from the hygromycinresistant embryos were transferred to a glasshouse and grown to maturity at $28^{\circ} \mathrm{C}$ under natural light conditions. Fourteen $\mathrm{T}_{0}$ plants transformed with pCN-Adh2 vector were produced from 130 introductions, in which one introduction corresponded to two bombardments per Petri dish containing approximately 20 embryo clusters. The first generated transgenic soybean was subjected to further analysis. Four $\mathrm{T}_{1}$ seeds were sown in a glasshouse and the $T_{1}$ soybean plants set $38,43,43$ and $44 \mathrm{~T}_{2}$ seeds, respectively. Twenty out of $38 \mathrm{~T}_{2}$ seeds which originated from one $\mathrm{T}_{1}$ plant were sown, and $\mathrm{T}_{2}$ seedlings were screened by PCR for inheritance of the Adh transgene. All 20 progenies of $\mathrm{T}_{2}$ soybean plants contained the Adh transgene according to PCR analysis. There were no segregated soybeans which did not

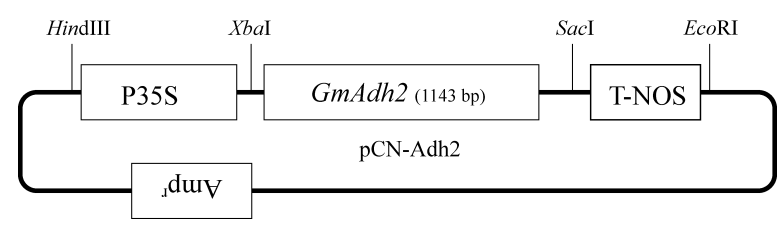

Figure 1. Structure of plasmid used for transformation. Plasmid pCN-Adh2 contains the sense GmAdh2 gene, which is driven by the 35S CaMV promoter.

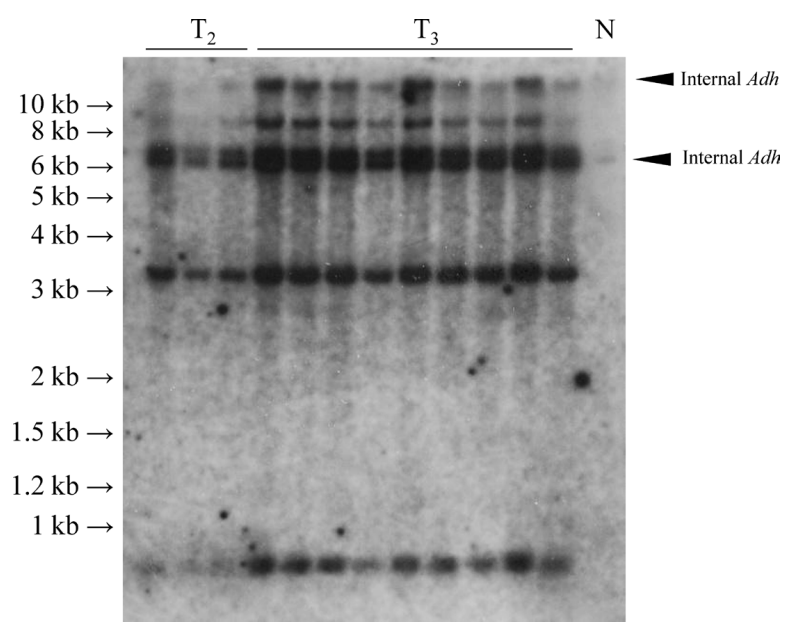

Figure 2. Southern blot hybridization analysis of $T_{2}$ and $T_{3}$ soybean plants in the same transgenic line. Genomic DNA $(5 \mu \mathrm{g})$ was digested with EcoRI, separated in $1 \%$ agarose gel, blotted onto the membrane, and hybridized with an Adh gene probe. 'N', non-transformed soybean (cv. Jack). 'Internal Adh' shows the estimated internal Adh gene that was originally contained in the non-transgenic soybean (cv. Jack).

contain the Adh transgene. Similarly, all $10 \mathrm{~T}_{3}$ progenies, which originated from one randomly selected abovementioned $\mathrm{T}_{2}$ plant, contained the Adh transgene. We performed Southern blot hybridization analysis with an Adh probe to characterize the insertion patterns in $\mathrm{T}_{2}$ and $\mathrm{T}_{3}$ soybeans (Figure 2). All $\mathrm{T}_{2}$ and $\mathrm{T}_{3}$ soybeans had the same EcoRI restriction fragments. Thus, the selected $\mathrm{T}_{2}$ soybeans were considered to be homozygous for the Adh transgenes.

To examine the changes of proteins in the transgenic soybeans, two-dimensional polyacrylamide gel electrophoresis (2-DE) was performed. Transgenic and non-transgenic soybean seeds were sterilized in a sodium hypochlorite solution, germinated on silica sand for 4 days, and subjected to protein extraction. Moreover, non-transgenic soybeans were germinated for 2 days and flooded with water for 2 days, and the soaking water was then drained before protein extraction. All growing was performed in a condition of white fluorescent light $\left(600 \mu \mathrm{mol} \mathrm{m}{ }^{-2} \mathrm{~s}^{-1}, 12 \mathrm{~h}\right.$ light period/day) at $25^{\circ} \mathrm{C}$ and $70 \%$ relative humidity in a growth chamber. Proteins were extracted from roots containing hypocotyls, separated by $2-\mathrm{DE}$, and detected by CBB staining. Protein analysis confirmed that Adh expression in the transgenic soybeans increased in comparison to non- 


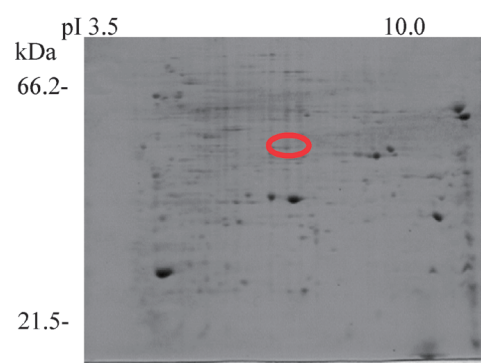

Without flooding stress

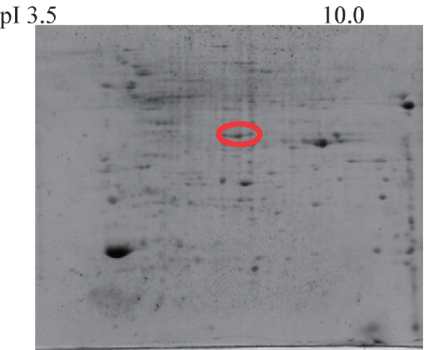

Flooding stress

Non-transgenic soybeans

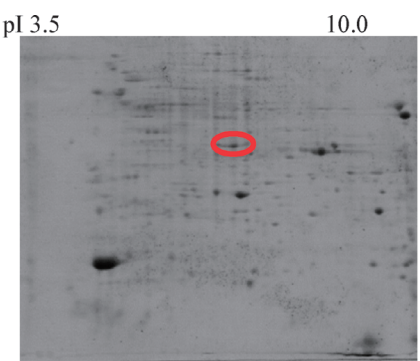

Without flooding stress

$\mathrm{T}_{3}$ soybean

with the $A d h$ transgene

Figure 3. 2-DE analysis of Adh protein expression in the roots and hypocotyls of non-transgenic soybeans and $\mathrm{T}_{3}$ soybeans with the Adh transgene. The left panel shows 2-DE analysis of non-flooded control seedlings at 4 days after sowing, and the middle panel shows 2-DE analysis of seedlings which were germinated for 2 days and flooded for 2 days. The right panel shows 2 -DE analysis of non-flooded $\mathrm{T}_{3}$ soybean seedlings at 4 days after sowing. The circle shows the position of Adh as confirmed by Komatsu et al. (2011).

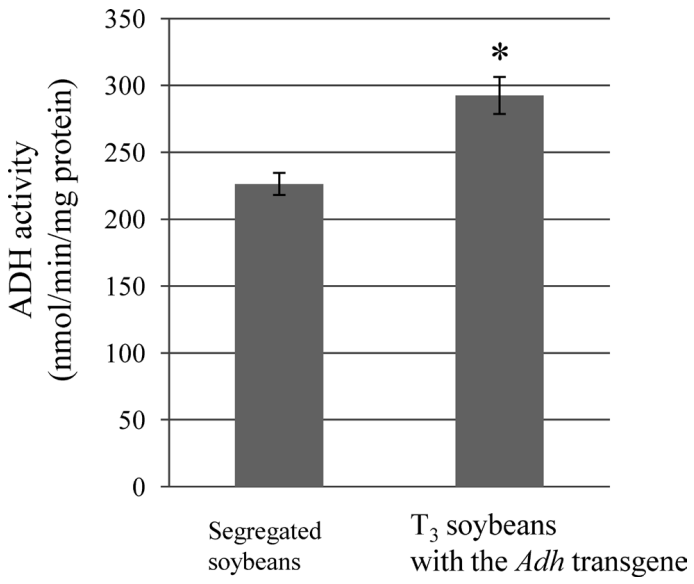

Figure 4. Adh activities in the roots and hypocotyls of $\mathrm{T}_{3}$ soybeans with the Adh transgene and transgene-segregated soybeans. The roots and hypocotyls of 4-day-old seedlings were collected. Adh activity was measured according to the method described by Nanjo et al. (2010). Values are the means $\pm \mathrm{SE}$ of germinated soybean seedlings. Asterisk indicates significance at $p<0.01$.

transgenic soybeans without flooding, and also increased as much as in non-transgenic soybeans subjected to flooding (Figure 3).

An assay for enzyme activity was performed to examine the change of Adh activity in the transgenic soybeans. $\mathrm{T}_{3}$ soybean seeds and segregated $\mathrm{T}_{2}$ soybean seeds that did not inherit the Adh transgene as controls were germinated for 4 days. Suspensions extracted from the roots and hypocotyls were subjected to an assay of Adh activity according to the method described by Nanjo et al. (2010). The Adh assay confirmed that Adh activities in the transgenic soybeans increased in comparison to control soybeans (Figure 4).

We subjected this transgenic soybean line to flooding conditions to investigate the response to flooding stress at the early stage of growth of the seedlings. Seeds of

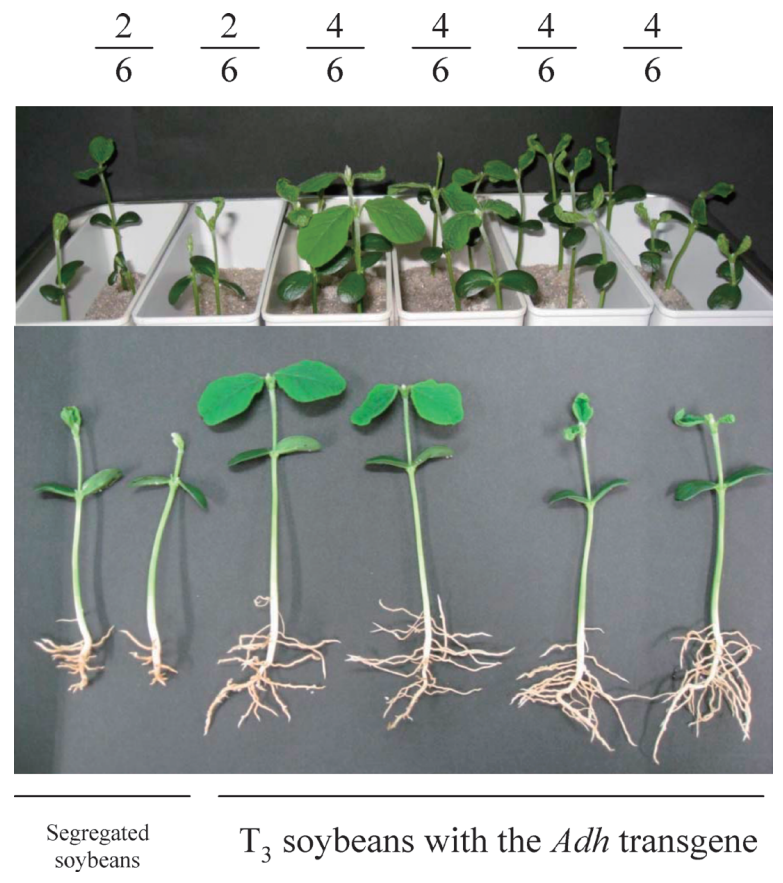

Figure 5. Growth responses of $\mathrm{T}_{3}$ soybeans with the Adh transgene and transgene-segregated soybeans after flooding. Soybean seeds were germinated on sand for 2 days and then treated with flooding for 4 days. The water was then removed, and the seedlings were allowed to develop until 14 days after sowing. The numbers on the upper part of the photograph show the germinated soybeans among 6 sown seeds.

transgenic soybeans were germinated for 2 days and flooded with water for 4 days, and the soaking water was then drained and the seedlings were allowed to grow. $T_{3}$ soybeans were supplied in quadruplicate with 6 seeds which each originated from different $\mathrm{T}_{2}$ soybeans. Six segregated $\mathrm{T}_{2}$ soybeans that did not inherit the Adh transgene were also subjected to flooding as controls. Figure 5 shows 9 days of growth after the water was drained. At a glance, the hypocotyls of the germinated 

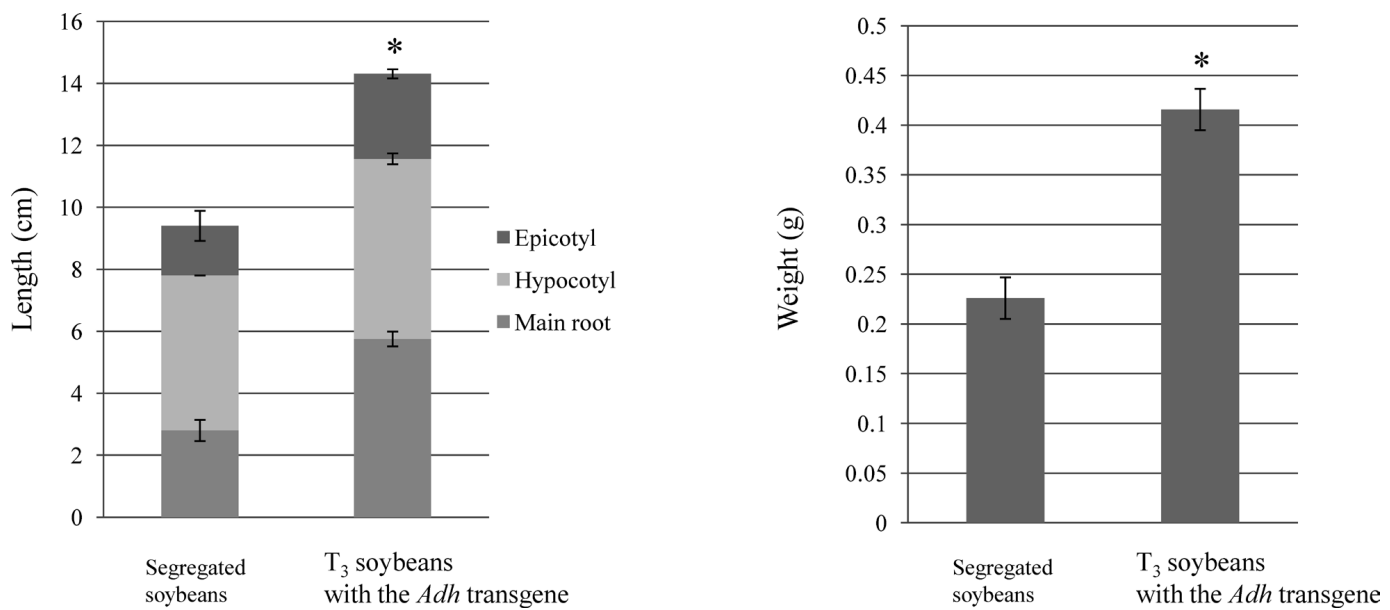

Figure 6. The seedling length and fresh weight of $\mathrm{T}_{3}$ soybeans with the Adh transgene and of transgene-segregated soybeans after flooding. The lengths of the soybean seedlings were calculated as the sum total of the main root, hypocotyl and epicotyl. Values are the means \pm SE of germinated soybean seedlings. Asterisk indicates significance at $p<0.01$.

control soybeans were thinner, the emerged primary leaves were smaller than those of transgenic soybeans, and the growth of the control soybeans was suppressed. Shi et al. (2008) reported that the total number of roots, the length of the main root, the length of the lateral and adventitious roots, and the fresh weight of the roots of flooded soybean seedlings were significantly suppressed compared with untreated plants after 3 days of flooding stress. A reduction in root growth is one of the most commonly reported parameters during flooding (Wang and Jiang 2007). Thus, we measured changes in the hypocotyl length, main root length, and total fresh weight. Morphological observation revealed that seedlings in the controls had shorter hypocotyls and main roots and a lighter total weight than $\mathrm{T}_{3}$ soybeans with the Adh transgenes (Figure 6). $\mathrm{T}_{3}$ soybeans also had more lateral roots than the control seedlings. The transgenic soybeans in this study showed greater tolerance of flooding in the early stage relative to the control soybeans.

Flooding leads to reduced gas exchange between the plant tissue and the atmosphere, because gases, particularly oxygen, diffuse 10,000 times more slowly in water than in air (Armstrong 1979). Oxygen deprivation by flooding is probably the primary signal triggering the response (Jackson and Colmer 2005), and it induces ethylene biosynthesis (Vriezen et al. 1999; Komatsu et al. 2009), cell wall loosening (Saab and Sachs 1996; Komatsu et al. 2010b), and aerenchyma formation (Thomas et al. 2005; Shimamura et al. 2010) in plants. Under limiting oxygen conditions, energy metabolism is shifted from oxidative to mainly alcohol fermentation (Smith and ap Rees 1979). Pdc and Adh catalyze alcohol fermentation, and are key enzymes for energy production under hypoxia conditions. Reduced flooding or hypoxic tolerances in the Adh null mutants of maize (Johnson et al. 1994), barley (Harberd and
Edwards 1982), Arabidopsis thaliana (Jacobs et al. 1988), and rice (Matsumura et al. 1995; 1998) have been reported. Thus, Adh is thought to play important roles in flooding anaerobic plant seed germination and growth. Some studies in which the Adh gene was overexpressed in plants were conducted to determine whether increased Adh activity would improve flooding tolerance. Rahman et al. (2001) demonstrated that transgenic rice with the cotton Adh2 gene showed no increase in survival following anoxia treatment, and higher Adh activities were not sufficient to increase the ability of rice to withstand anoxia. However, F1 plants containing both the $P d c$ and $A d h$ genes showed increased anoxia tolerance. Similarly, Ismond et al. (2003) found that overexpression of Arabidopsis Adh1 in transgenic Arabidopsis had no effect on flooding survival, but overexpression of Arabidopsis Pdc1 or Pdc2 resulted in improved plant survival. The expression of Pdc or Adh and its contribution to the plant's ability to survive flooding might depend on the carbon flow and ethanol fermentation of the plant species. In this study, we introduced the GmAdh2 gene to soybeans and subjected the soybeans to flooding stress. The first acquired line of transgenic soybean seedlings germinated vigorously relative to control soybeans after flooding. Protein analysis confirmed that Adh in the transgenic soybeans was up-regulated more than that in non-transgenic soybeans. Moreover, Adh activities in the transgenic soybeans increased in comparison to control soybeans. It was assumed that constitutive expression of Adh in transgenic soybeans led to an alteration of carbon flow along with glycolysis and ethanol fermentation under flooding low oxygen stress conditions, and consequently brought about vigorous germination. Hereafter, it will be necessary to investigate the fluctuations of glycolysis, fermentation enzymes, and substrates on the carbohydrate metabolic pathways in transgenic soybeans, 
and to search for flood-tolerant transgenic soybeans among the 13 transgenic soybean lines that remained unexamined in this report. This study is the first report in which the $A d h$ gene was transformed in soybeans and in which flooding stress responses were investigated in this transgenic soybean.

\section{Acknowledgements}

We thank Motoko Kobayashi (NARO Agricultural Research Center for the Tohoku Region, NARCT) for technical assistance. We also thank Dr. Masao Ishimoto (National Institute of Agrobiological Sciences, NIAS) for useful advice. This work was funded by grants from the National Agriculture and Food Research Organization, Japan.

\section{References}

Armstrong W (1979) Aeration in higher plants. Adv Bot Res 7: 225-232

Furutani N, Hidaka S (2004) Efficient production of transgenic soybean using a co-transformation method. Breed Sci 54: 91-98

Harberd NP, Edwards KJR (1982) The effect of a mutation causing alcohol dehydrogenase deficiency on flooding tolerance in barley. New Phytol 90: 631-644

Hashiguchi A, Sakata K, Komatsu S (2009) Proteome analysis of early-stage soybean seedlings under flooding stress. J Proteome Res 8: 2058-2069

Ismond KP, Dolferus R, Pauw MD, Dennis ES, Good AG (2003) Enhanced low oxygen survival in arabidopsis through increased metabolic flux in the fermentative pathway. Plant Physiol 132: 292-1302

Jackson MB, Colmer TD (2005) Response and adaptation by plants to flooding stress. Ann Bot (Lond) 96: 501-505

Jacobs M, Dolferus R, Van Den Bosshe VB (1988) Isolation and biochemical analysis of ethyl methyl sulfonate induced alcohol dehydrogenase null mutants of Arabidopsis thaliana (L.) Heynth. Biochem Genet 26: 102-112

Johnson JR, Cobb BG, Drew MC (1994) Hypoxic induction of anoxia tolerance in roots of Adh1 null Zea mays L. Plant Physiol 105: 61-67

Komatsu S, Yamamoto R, Nanjo Y, Mikami Y, Yunokawa H, Sakata K (2009) A comprehensive analysis of the soybean genes and proteins expressed under flooding stress using transcriptome and proteome techniques. J Proteome Res 8: 4766-4778

Komatsu S, Sugimoto T, Hoshino T, Nanjo Y, Furukawa K (2010a) Identification of flooding stress responsible cascades in root and hypocotyl of soybean using proteome analysis. Amino Acids 38: 729-738

Komatsu S, Kobayashi Y, Nishizawa K, Nanjo Y, Furukawa K (2010b) Comparative proteomics analysis of differentially expressed proteins in soybean cell wall during flooding stress. Amino Acids 39: 1435-1449
Komatsu S, Deschamps T, Hiraga S, Kato M, Chiba M, Hashiguchi A, Tougou M, Shimamura S, Yasue H (2011) Characterization of a novel flooding stress-responsive alcohol dehydrogenase expressed in soybean roots. Plant Mol Biol 77: 309-322

Matsumura H, Takano T, Yoshida KT, Takeda G (1995) A rice mutant lacking alcohol dehydrogenase. Breed Sci 45: 365-367

Matsumura H, Takano T, Takeda G, Uchimiya H (1998) Adh1 is transcriptionally active but its translational product is reduced in a rad mutant of rice (Oryza sativa L.), which is vulnerable to submergence stress. Theor Appl Genet 97: 1197-1203

Nakayama N, Hashimoto S, Shimada S, Takahashi M, Kim YH, Oya T, Arihara J (2004) The effect of flooding stress at the germination stage on the growth of soybeans in relation to initial seed moisture content. Jpn J Crop Sci 73: 323-329, (in Japanese)

Nanjo Y, Skultety L, Ashraf Y, Komatsu S (2010) Comparative proteomic analysis of early-stage soybean seedlings responses to flooding by using gel and gel-free techniques. J Proteome Res 9: 3989-4002

Newman KD, Vantoai TT (1992) Molecular characterization of the soybean alcohol dehydrogenase gene family amplified in vitro by the polymerase chain reaction. Plant Physiol 100: 489-495

Rahman M, Grover A, Peacock WJ, Dennis ES, Ellis MH (2001) Effects of manipulation of pyruvate decarboxylase and alcohol dehydrogenase levels on the submergence tolerance of rice. Aust J Plant Physiol 28: 1231-1241

Rees T, Jenkin LET, Smith AM, Wilson PM (1987) The metabolism of flood-tolerant plants. In: Crawford RMM (ed) Plant Life in Aquatic and Amphibious Habitats. Blackwell Scientific Publications, Oxford, pp 227-238

Russell DA, Wong DML, Sachs MM (1990) The anaerobic response of soybean. Plant Physiol 92: 401-407

Saab IN, Sachs MM (1996) A flooding-induced xyloglucan endotransglycosylase homolog in maize is responsive to ethylene and associated with aerenchyma. Plant Physiol 112: 385-391

Shi F, Yamamoto R, Shimamura S, Hiraga S, Nakayama N, Nakamura T, Yukawa K, Hachinohe M, Matsumoto H, Komatsu S (2008) Cytosolic ascorbate peroxidase 2 (cAPX 2) is involved in the soybean response to flooding. Phytochemistry 69: $1295-1303$

Shimamura S, Yamamoto R, Nakamura T, Shimada S, Komatsu S (2010) Stem hypertrophic lenticels and secondary asrenchima enable oxygen transport to roots of soybean in flooded soil. Ann Bot (Lond) 106: 277-284

Smith AM, ap Rees T (1979) Pathways of carbohydrate fermentation in the roots of marsh plants. Planta 146: 327-334

Thomas AL, Guerreiro SM, Sodek L (2005) Aerenchyma formation and recovery from hypoxia of the flooded root system of nodulated soybean. Ann Bot (Lond) 96: 1191-1198

Vriezen WH, Hulzink R, Mariani C, Voesenek LA (1999) 1-aminocyclopropane-1-carboxylate oxidase activity limits ethylene biosynthesis in Rumex palustris during submergence. Plant Physiol 121: 189-196

Wang K, Jiang Y (2007) Antioxidant responses of creeping bentgrass roots to water-logging. Crop Sci 47: 232-238 